\title{
Tsafon
}

Revue d'études juives du Nord

$75 \mid 2018$

Exil des langues - Langues d'exil

\section{Claude Vigée : exil des langues sous la « lune d'hiver »}

Andrée Lerousseau

\section{OpenEdition}

1 Journals

Édition électronique

URL : https://journals.openedition.org/tsafon/567

DOI : $10.4000 /$ tsafon. 567

ISSN : 2609-6420

Éditeur

Association Jean-Marie Delmaire

Édition imprimée

Date de publication : 1 juin 2018

Pagination : 43-54

ISSN : 1149-6630

\section{Référence électronique}

Andrée Lerousseau, «Claude Vigée : exil des langues sous la «lune d'hiver » », Tsafon [En ligne], 75|

2018, mis en ligne le 06 mai 2019, consulté le 24 juin 2021. URL : http://journals.openedition.org/ tsafon/567 ; DOl : https://doi.org/10.4000/tsafon.567

Tsafon. Revues d'études juives du Nord 


\title{
Claude Vigée : exil des langues sous la « lune d'hiver »
}

\author{
Andrée Lerousseau* \\ Je t'ai porté longtemps sous l'écorce des pluies \\ Dans la maternité virile de l'attente ${ }^{1}$
}

Évoquant le chemin parcouru par Claude Vigée, Stéphane Mosès remarquait: «Plus profondément que dans l'alternance des lieux, que dans la succession des exils et des retours, cette trajectoire s'inscrit sans doute dans les errances du langage $»^{2}$. D'où cette « hantise de Babel $»^{3}$ qui, selon Sylvie Parizet, habite l'écrivain, victime, dès sa plus tendre enfance d'une «expropriation linguistique» ${ }^{4}$ vécue comme une mutilation. La succession et l'alternance des lieux, s'accompagnant de « l'emmêlement des langues inlassables/ Qui jacassent le long des fils de notre errance ", contribuera à renforcer cet état de confusion et de désorientation et le sentiment que «Toute terre est exil,/ Toute langue étrangère », sans parvenir toutefois à entamer «l'espoir en la parole »

\footnotetext{
*Université de Lille, SHS.

${ }^{1}$ Claude Vigée, "La Pâque du roi Josias », dans La Corne du Grand Pardon (19511954), Mon heure sur la terre. Poésies complètes, 1936-2008 [désormais MHT], préface de Michèle Finck, introduction d'Anne Mounic, Paris, Galaade Éditions, 2008, p. 203.

${ }^{2}$ Stéphane Mosès, "Errances du langage. De l'origine perdue à l'origine retrouvée », dans L'œil témoin de la parole, essais réunis par David Mendelson et Colette Leinmann, avec des textes inédits de Claude Vigée, Paris, Parole et Silence, 2001, p. 101.

${ }^{3}$ Claude Vigée, Sylvie Parizet, Les portes éclairées de la nuit. Entretiens, essais, cahier, récits inédits (2000-2006), Paris, Cerf, 2006, p. 133.

${ }^{4}$ Claude Vigée, La lune d'hiver. Récit-Journal-Essai, Paris, Champion, 2002, p. 244.
} 
comme « promesse du monde $»^{5}$. Il est en effet une ruse de l'exil, calquée sur le modèle de celle de la raison chez Hegel, « ennemi intime » de Vigée :

Rusé paradoxe de l'exil ! Il se veut à la fois le site du tourment, de la tentation et de la révélation. La souffrance est aussi un royaume de l'homme, l'absence une présence, et l'exil un foyer... ${ }^{6}$

Cela vaut en particulier pour les années passées en Amérique, de 1942 à 1960, sous la lumière indécise et blafarde de la lune d'hiver, « cette obscurité blanche et pétrifiante qui n'est ni nuit ni jour. Lumière d'une conscience appauvrie et diminuée ${ }^{7}$, à l'opposé de la lumière de Judée, mais qui n'en demeure pas moins une période de « germination du grain [...] dans la nuit, le silence de la terre frappée par le long gel hivernal », dont Goethe déjà aurait confié le secret au jeune lycéen de Strasbourg ${ }^{8}$.

Après un rappel du rapport entretenu par Claude Vigée aux langues tout au long d'un itinéraire entre exils et retours, nous porterons une attention particulière à cette longue période d'hibernation, mais également de gestation, durant laquelle il s'affirmera comme l'un des grands poètes juifs de langue française, avant de devenir, selon le jugement de Robert Misrahi, le fondateur de la littérature israélienne de langue française ${ }^{9}$. Cela nous conduira à analyser la spécificité de la langue de Vigée dans laquelle coïncide «l'écriture de l'exil et l'exil de (dans) l'écriture, au point », ajoute Jean-François Chiantaretto dans son introduction à La lune d'hiver, «que la fin de l'exil diasporique et l'apprentissage de la langue originaire (l'hébreu) ne changeront rien à cet

\footnotetext{
${ }^{5}$ C. Vigée, Le poème du retour (1960-1961), dans MHT, op. cit., p. 384, 381 et 389.

${ }^{6}$ Claude Vigée, La faille du regard. Essais et entretiens, Paris, Flammarion, 1987, p. 235. La seconde phrase reprend deux vers du poème « Je ne nie pas la nuit, la trop aimée... » dans L'été indien, dans MHT, op. cit., p. 293. Un passage de La lune d'hiver précise toutefois que ce foyer «n'est qu'un feu de camp provisoire» (p. 234). Concernant Hegel comme « ennemi intime » de Vigée, cf. son entretien avec Stéphane Mosès, rappelé par ce dernier dans «L'esthétique de la présence », dans Hélène Péras et Michèle Finck (dir.), La Terre et le Souffle. Rencontre autour de Claude Vigée, 22-29 août 1988, Paris, Albin Michel, 1992, p. 213.

${ }^{7}$ Claude Vigée, Les orties noires flambent dans le vent (Un requiem alsacien), Paris, Flammarion, 1894, p. 88.

${ }^{8}$ C. Vigée, La faille du regard, op. cit., p. 277.

${ }^{9}$ Robert Misrahi, «À propos de La lune d'hiver» (1ère parution dans La Quinzaine littéraire en septembre 1970), Peut-être $\mathrm{n}^{\circ} 4,2013$, p. 55 : «la littérature israélienne de langue française (dont Claude Vigée est proprement le créateur) ».
} 
égard $»^{10}$. «Vêtement bien tissé dans la chair vive de l'âme » ${ }^{11}$, le français accompagnera le poète dans toutes ses pérégrinations.

\section{« Les errances du langage »}

Né à Bischwiller, petite ville de Basse-Alsace, en janvier 1921, Claude-André Strauss patauge, enfant, dans «une vraie mare aux grenouilles linguistique », " un bouillon de haute culture philologique $»^{12}$ où coexistent le yiddish-daïtsch, judéo-alsacien, le goïmlisch, dialecte bas-rhinois des Gentils issu du Mittelhochdeutsch et commun, en dépit de ses variantes, à l'ensemble des habitants d'Alsace, le français du dimanche, fraîchement sorti de la naphtaline quand il y avait de la visite, et l'allemand livresque. Puis survint, en octobre 1926, la terrible révélation faite aux enfants par leur maîtresse d'école : «-e Lapin isch $e$ Haas ! $»^{13}$. Ainsi débuta le premier exil, hors de la langue maternelle frappée d'interdit «au nom d'une idéologie jacobine et centralisatrice hostile à tout régionalisme ${ }^{14}$ : « Marqués au fer rouge du dépaysement linguistique, et flagellés d'orties, nous subîmes à notre corps défendant une initiation à l'exil, au sein même de notre pays natal », se souvient Vigée qui ne verra d'autre remède à la stigmatisation et à la blessure psychique infligées que $d$ '«engendrer en [lui] un nouvel être de langage » en devenant poète ${ }^{15}$.

Si le jeune garçon ressent la langue imposée comme un bâillon, un obstacle à la spontanéité et à l'expression de l'intériorité, il n'en pressent pas moins que l'acquisition du français, langue de culture, policée, structurée, permettant de tisser des liens, d'établir des rapports, est la condition d'une ouverture sur l'avenir et sur le monde intellectuel :

Ce qui m'a sauvé du mutisme intellectuel, c'est d'apprendre le français. Sinon, je serais resté sur le modèle mythique de mon aïeul Léopold, une sorte de primitif de la parole, un homme de l'archaïsme rural, menacé par l'aphasie originelle. ${ }^{16}$

\footnotetext{
${ }^{10}$ C. Vigée, La lune d'hiver, op. cit., introduction, p. 15.

${ }^{11}$ C. Vigée, Les orties noires, op. cit., p. 85-86.

${ }^{12}$ Claude Vigée, Rêver d'écrire le temps, de la forme à l'informe, Paris, Orizons, 2011, p. 533.

${ }^{13}$ C. Vigée, La lune d'hiver, op. cit., p. 250 : «- un lapin, c'est un Haas ».

${ }^{14} \mathrm{~S}$. Mosès, «Errances du langage de l'origine perdue à l'origine retrouvée », op. cit., p. 101.

${ }^{15}$ C. Vigée, Les orties noires, op. cit., p. 9 et 81.

${ }^{16}$ C. Vigée, S. Parizet, Les portes éclairées de la nuit, op. cit., p. 99.
} 
Et le voici bientôt qui "patine» sur la langue, obéissant à ses mouvements rythmiques, attentif «à la qualité ontique des voyelles irisées [...] qu'enchâssaient les consonnes sévèrement disposées à l'entour », au " rayonnement sonore » de certains mots et au souffle, à la respiration ${ }^{17}$. Mais le patineur n'est-il pas précisément celui qui glisse sur la surface des choses, symbole ou figure d'une certaine superficialité ? N'y a-t-il pas là une trop grande légèreté de la parole, dont il lui faudra se déprendre?

Il nous faut [...] nous défendre en quelque idiome que ce soit [...] de la brillante gymnastique des marionnettes verbales. Cette menace n'est que trop évidente en français, vieille langue de culture, glissante, articulée, affinée et usinée à l'excès, soumise à un polissage formel qui, en des mains sans scrupules, fait d'elle l'instrument de prestidigitations intellectuelles coupées des racines sensibles, seules authentiques. ${ }^{18}$

C'est à cette tentation des belles figures imposées qu'il lui faudra résister lorsque, au cœur de l'hiver américain, le français s'imposera à lui comme langue d'écriture et de poésie. Entretemps, Claude-André Strauss, confronté à l'instar de nombre d'Alsaciens à l'exode et en tant que Juif à l'exclusion et à la trahison de sa patrie ${ }^{19}$, sera devenu Claude Vigée, pseudonyme sous lequel il publie un poème dans Poésie 42, revue dirigée par Pierre Seghers, et qui, en écho au 'haï ani, au « moi vivant» de la prophétie d'Isaïe (49-18), résonne comme un véritable défi lancé aux bourreaux et à leurs collaborateurs ${ }^{20}$. Membre d'un groupe de militants se rassemblant dans la vieille synagogue de la rue Palaprat à Toulouse, où il s'est réfugié et poursuit des études de médecine, le jeune homme renoue avec ses racines et entre en résistance au sein de l'Action

\footnotetext{
${ }^{17}$ Ibid., p. 99, 116-117 où Vigée évoque « la symphonie verbale somptueuse, rutilante et baroque » du prélude d'Atala, et p. 120.

${ }^{18}$ C. Vigée, La lune d'hiver, op. cit., p. 247-248.

${ }^{19} \mathrm{Sa}$ vie bascule le 19 octobre 1940 lorsqu'il découvre le «statut des Israélites » en première page de Paris-Soir: «Je demeurai debout quelques minutes sur la place ensoleillée, en proie à une sorte d'étourdissement, le souffle coupé de rage et d'indignation impuissantes. Il me semblait que j'avais été frappé d'un coup en plein cœur. Jamais je n'ai oublié, jamais je n'oublierai cet instant-là. Il a achevé de diviser ma vie en deux temps irréconciliables: celui de la confiance, celui du doute et de l'abandon. Les versants de mon existence déchirée ne se rejoindraient plus ici-bas. » (dans La lune d'hiver, op. cit., p. 13).

${ }^{20}$ Voir à ce propos dans le recueil L'Été indien la strophe du poème «Épines et chardons » intitulée " 'Hayani» : « Mon amour est la terre,/ Le soleil mon mystère,/ Ma parole de vent -/ Mon nom : Je suis vivant. » (dans MHT, op. cit., p. 369).
} 
juive. Le 26 octobre 1942, alors que l'étau se resserre autour de lui, il franchit avec sa mère la frontière des Pyrénées et tous deux s'embarquent le 19 novembre à Lisbonne sur le Serpa Pinto faisant route vers New York. Dans le Nouveau Monde, où il retrouve Évy, sa cousine, qu'il épouse en 1947, et où naissent leurs deux enfants, Claude Vigée, malgré une brillante carrière de comparatiste à l'Université de Brandeis, à proximité de Boston, et une reconnaissance internationale de son œuvre de poète, saluée par Saint-John Perse, Camus et tant d'autres, se sent « out of place $»^{21}$.

Un adjectif plus que tout autre résume à lui seul la nature de ce monde : c'est, dit Vigée, « un monde incirconcis ${ }^{22}$, déserté par la présence, où aucune alliance ne se noue, pas même entre les hommes (il n'y a ni prochains, ni cercle de voisins ${ }^{23}$ ), au sein duquel les êtres sont interchangeables, un monde de pénurie profonde en dépit de ses excès, sans passé, ni avenir, uniquement fait «d'intermittences ${ }^{24}$, hostile aux engendrements et interdisant tout accroissement de l'être, un univers de morts-vivants, à l'inverse du monde de l'enfance et de la Terre promise :

Monde où rien n'arrive, où rien ne s'engendre ni ne s'enchaîne pour devenir davantage soi-même, où tout d'avance survit et où cela même qui n'est pas encore enfanté s'enlise dans son marasme à venir. ${ }^{25}$

Loin d'être perçu comme une ouverture et un recul à l'infini de l'horizon, l'espace américain apparaît au contraire comme un «mauvais infini ${ }^{26}$, et le poète confie qu'il se sent limité par tant d'immensité et comme « incarcéré du dehors ${ }^{27}$.

Un même déficit affecte la langue qui semble à Claude Vigée " surajoutée », comme une greffe qui ne prend pas. Incapable d'exprimer, voire d'atteindre même son être profond, elle contribue à éveiller et à renforcer en lui le sentiment d'une dissonance entre sa vie intérieure et son environnement, et constitue paradoxalement une obstruction à la parole :

\footnotetext{
${ }^{21}$ C. Vigée, La lune d'hiver, op. cit., p. 233.

${ }^{22}$ Ibid., p. 150.

${ }^{23}$ Ibid., p. 236. Sur la présence des voisins caractéristique du monde de l'enfance, voir l'article d'Alfred Dott, «Un monde fait exclusivement de voisins, une vie sans aucune distance... ", Peut-être n ${ }^{\circ} 1,2010$, p. 30-31.

${ }^{24}$ C. Vigée, La lune d'hiver, op. cit., p. 148.

${ }^{25}$ R. Misrahi, «À propos de La lune d'hiver», op. cit., p. 148.

${ }^{26}$ C. Vigée, Les orties noires, op. cit., p. 98.

${ }^{27}$ C. Vigée, La lune d'hiver, op. cit., p. 214 et p. 150 pour la citation.
} 
L'Amérique m'a été extrêmement hospitalière, bonne, mais elle m'est restée étrangère $[\ldots]$ Je crois que la question de la langue jouait un très grand rôle. J'en suis devenu conscient plus tard. La langue que j'étais bien obligé d'adopter, l'anglais, me paraissait surajoutée. J'avais comme un bâillon intérieur. Je parlais librement, mais ce qui parlait en moi, ce n'était pas le fond de mon être, c'était une contrefaçon [...] ne pouvant vraiment dire ce que je sentais au fond de moi, mon chemin lui-même était obstrué. ${ }^{28}$

Et de nouveau, comme lors du passage forcé au français et de l'abandon du dialecte natal, naît l'impression d'une amputation du vivant :

Je me sentais mutilé avec l'anglais [...] Je ne pouvais pas, en anglais, m'incarner de façon shameless [...] Je suis quelqu'un qui aime à être shameless. J'avais le sentiment que je ne pouvais pas, en anglais, m'ébattre. ${ }^{29}$

Le langage de la spontanéité et de l'authenticité dans lequel l'être autrefois s'incarnait et s'ébattait librement et sans honte, la langue de l'enfance, demeure refoulée dans l'inconscient, frappée d'un exil redoublé : « les noyaux pulsants originels de l'âme restent [...] ensevelis sous les strates de glace et de cendres d'un monde indifférent, qui les nie ou les étouffe d'instinct», comme « submergés par le doute, le sentiment d'inutilité et d'impuissance créatrice $»^{30}$. Le dialecte natif ne se risque à la parole que sous le masque de l'allemand :

Aux moments des plus grandes crises de mon existence, le dialecte ou bien ses masques d'emprunts, ses succédanés en haut allemand, ont chaque fois resurgi chez moi pour exprimer, sans entraves inutiles, le désarroi de l'heure [...] C'est à travers certaines réminiscences allusives, quelques copeaux de parole légers, quelques débris de souvenirs échappés à la Hochsprache littéraire apprise jadis au collège [...] que s'effectuait le travail clandestin de la mémoire bloquée ! ${ }^{31}$

\footnotetext{
${ }^{28}$ Claude Vigée, Danser vers l'abîme ou la spirale de l'extase. Choix de poèmes et d'essais, 1995-2004, Paris, Parole et Silence, 2004, p. 204.

${ }^{29}$ Claude Vigée, Mélancolie solaire. Nouveaux essais, cahiers, entretiens inédits, poèmes (2006-2008), avant-propos et édition d'Anne Mounic, Paris, Orizons, 2008, p. 84.

${ }^{30}$ C. Vigée, La faille du regard, op. cit., p. 269 et 276.

${ }^{31}$ Ibid., p. 278-277. Ainsi par exemple dans le poème «Winterweiden », évoquant des saules roidis, pétrifiés par le gel, symboles d'une attente insensée et d'une absence au monde (dans La Corne du Grand Pardon, MHT, op. cit., p. 181). Notons toutefois dans le recueil suivant, L'été indien, une brève résurgence de la langue natale dans une comptine, en exergue au poème «Au vent bleu du couchant» (ibid., p. 308).
} 
Le haut allemand scolaire permettait au poète à la fois de garder la distance causée par l'interdit du dialecte et de s'en rapprocher :

\begin{abstract}
À la fois proches et lointains, ces moments musicaux en Hochdeutsch d'emprunt jaillissaient en Amérique du fond de ma sensibilité, telles les froides étincelles d'un monde révolu, renvoyées à la surface de ma conscience d'exilé explorée à l'aide d'un miroir grossissant; mais aussi, peut-être, comme les brefs éclairs bleus annonciateurs d'une improbable reconquête future de mon identité totale, morcelée par l'histoire de notre siècle. ${ }^{32}$
\end{abstract}

L'heure du grand retour et de la reconquête de soi et de son identité sonne en 1960, lorsque Claude Vigée se voit offert un poste à l'Université hébraïque de Jérusalem :

Le cœur qui sut attendre au plus noir de l'exil

Et survivre à minuit, par la grâce de l'agonie

Connaîtra la nouvelle joie qui germait dans le temps. ${ }^{33}$

Dans l'éblouissement de la lumière de Judée, il fait l'expérience d'une nouvelle naissance et d'une alliance nouvelle partagée avec le peuple et la femme aimée dans les « Noces d'Amnon et de Tamar ${ }^{34}$, chant jubilatoire et expression de cette plénitude, de cette extase à la fois spirituelle et sensuelle à laquelle il aspirait. À quarante ans, il apprend aux côtés de son fils l'hébreu élémentaire. S'il ne parviendra pas à s'assujettir la langue originaire au point d'en faire un instrument de création littéraire, l'hébreu exercera une influence considérable sur son œuvre poétique et poétologique, venant confirmer ses intuitions : ainsi par exemple celle « du bon en avant inconditionnel $»^{35}$ en direction d'un futur inouï, illustré selon lui dans le «vav conversif» de la grammaire hébraïque, "procédé métaphysique révolutionnaire qui inverse le passé en futur et le futur en passé, le courant négatif en positif, et rend leur alternance possible » et substitue au «temps fatigué, usé, dévalué » (le temps de l'exil) « un temps vivant » jetant un pont vers l'avenir ${ }^{36}$.

\footnotetext{
${ }^{32}$ C. Vigée, La faille du regard, op. cit., p. 276-277.

${ }^{33}$ « Le poème du retour », dans $M H T$, op. cit., p. 379. La « montée vers Jérusalem » est vécue comme une sortie d'Égypte (cf. Les orties noires, op. cit., p. 106-107).

${ }^{34}$ Dans MHT, op. cit., p. 390-398.

${ }^{35}$ C. Vigée, La faille du regard, op. cit., p. 43.

${ }^{36}$ Claude Vigée, L'extase et l'errance, Paris, Grasset, 1982, p. 35 ; et La faille du regard, op. cit., p. 216.
} 
Et comment ne pas songer à l'alphabet hébraïque, vecteur de la création, dans le souhait exprimé par Vigée en 1987 dans La faille du regard:

[...] j'ai voulu que la lettre soit le tremplin vers le lendemain qui parle - pas seulement la trace avarement résumée, ou l'inscription funéraire, d'un passé révolu [...] la lettre ouverte, décachetée, doit se sentir [...] lourde du futur inouï, enceinte de l'avent, de l'événement imprévisible. ${ }^{37}$

Dans la langue originaire (l'hébreu), il retrouve également la matérialité et la saveur un peu âpre de la langue originelle qui, par un processus d'anamnèse, refait surface en été 1982, durant la guerre du Liban : l'angoisse éprouvée à la pensée de ses étudiants engagés sur le front et le danger qui pèse sur la nouvelle génération ravivent en Vigée le souvenir d'une génération de jeunes Alsaciens «vouée tout entière au désastre $»^{38}$ et, sur sa terrasse, à Jérusalem, il compose en quelques jours le « requiem alsacien », Schwàrzi sengessle flàckere ém Wénd [Les orties noires flambent dans le vent], bientôt suivi d'un second recueil, Le feu d'une nuit d'hiver, également rédigé en dialecte.

« Finalement », conclut Robert Misrahi en évoquant le long séjour sous la lune d'hiver :

[...] L'Amérique d'exil chasse encore, par l'intérieur, le poète vers la seconde et ultime médiation de son destin : Israël. C'est en Israël que se trouve le peuplepoète, le peuple conscient d'être ancré sur la plus ancienne origine, soucieux, comme le poète, de la synthèse entre l'être substantiel et l'originaire. ${ }^{39}$

\section{Un poète juif de langue française}

Évoquant l'exil aux États-Unis, Claude Vigée constate : «Ce long hivernage n'a pas réussi à me faire rentrer l'air dans la gorge ${ }^{40}$. Creusant sous les décombres amassés en lui depuis l'enfance jusqu'à parvenir à cette «nappe phréatique du commencement intime » qu'il nomme «le lac de la rosée », le domaine silencieux de l'Aleph où s'origine la création, «source de toutes les paroles articulées [...] qui

\footnotetext{
${ }^{37}$ Ibid., p. 217 et 218.

${ }^{38}$ Voir la citation de La lune d'hiver, en exergue aux Orties noires.

${ }^{39}$ R. Misrahi, « À propos de La lune d'hiver», op. cit., p. 55.

${ }^{40}$ C. Vigée, La faille du regard, op. cit., p. 280.
} 
adviennent en nous plus tard au cours de notre pérégrination terrestre $\gg{ }^{41}$, le poète frayera un passage au vivant, à la parole articulée en français. Dans une "mise en forme du silence en son, du son en parole, de la parole à la prière et de la prière au chant», selon les quatre étapes dégagées par Colette Leinmann dans son commentaire du poème de L'été indien intitulé « Destin du poète ${ }^{42}$, il fera parler l'autre en lui, tout en mettant à jour et à nu son être, cette partie intime demeurée intacte au sein même de l'exil, dans une double conjuration de la présence et du " paraître de l'être qui s'est dérobé » ${ }^{43}$ qui constitue l'essence même de l'art et de la poésie. Mais la rencontre de la réalité de soi et de l'autre en soi s'accompagne du dévoilement de la réalité du monde, conformément à la définition que donne Schelling de la mission de l'artiste pour lequel « représenter le contenu infini dans sa forme la plus parfaite » signifie le représenter dans sa forme « la plus chargée de finitude » ${ }^{44}$. Pas question, en effet, pour Vigée, de se réfugier dans l'ascèse ou de hisser le chant dans la sphère sublimité : l'assomption du chant, le passage du vivant se fait au forceps, à travers l'opacité du temps, fût-ce pour jaillir hors du temps.

Mais encore faut-il pour cela ressusciter la langue afin de l'extraire de son exil. En effet, « toute langue est exil! Si nous ne réussissons pas à la vivifier, à la greffer en nous, sur le lieu profond du jaillissement premier [...], nous végétons dans un lieu funéraire ${ }^{45}$. Il faut faire passer sur elle le souffle-esprit, le rua' $h$, en nous servant de notre corps. Tel est l'enjeu du recueil La Corne du Grand Pardon, rédigé entre 1951 et 1954, auquel le souvenir d'Ézéchiel et de l'épisode dans la vallée des ossements confère sa structure : à une description de la vallée dans la première partie succède le questionnement («Ces ossements vivrontils ?») et le miracle («Les ossements s'assemblèrent») débouchant sur la célébration de «l'arbre de vie». Ainsi le poète qui a choisi de s'exprimer en français, langue à la syntaxe rigide dont Rousseau a souligné le caractère apoétique, langue carcan tendant à la momification, va-t-il devoir donner rythme et mouvement à «ces articulations

\footnotetext{
${ }^{41}$ Claude Vigée, Le silence de l'Aleph. Écriture et Révélation, Paris, Albin Michel, 1992, p. 153. De cette eau, Claude Vigée dira qu'elle seule «désaltère l'errant du désert » (dans Mélancolie solaire, op. cit., p. 248).

${ }^{42}$ Colette Leinmann, "'Le Toi silencieux qui se parle en moi-même'», dans L'œil témoin de la parole, op. cit., p. 146 et 147.

${ }^{43}$ C. Vigée, La lune d'hiver, op. cit., p. 294.

${ }^{44}$ Ibid., cité p. 201.

${ }^{45}$ C. Vigée, Rêver d'écrire le temps, op. cit., p. 539.
} 
sclérosées » et conférer une saveur nouvelle à un idiome trop codifié et formalisé ${ }^{46}$.

Cette résurrection passe par la mémoire, y compris charnelle, de la langue première, celle de l'enfance, par ce « tremplin irremplaçable pour le saut dans l'inconnu, le plongeon dans l'abîme du temps futur », audelà de l'exil, par le «Sprungbrett matriciel de l'Alsace dialectale »" ${ }^{47}$, suivant l'alchimie propre à la fabrication du « Nusseschnaps », de la liqueur de noix fabriquée par le grand-père Léopold, à laquelle il est fait référence dans La lune d'hiver :

Pour fabriquer un litre de «Nusseschnaps » il faut de l'eau-de-vie et quelques noix fraîches [...] L'alcool filtre lentement à travers écales et coquilles, en se chargeant de leurs substances. De cette osmose naîtra l'exquise liqueur... L'alcool : la langue française, limpide, pénétrante, analytique. La noix opaque gaulée sur l'arbre, fruit de la terre nourricière, y macère dans son brou [...] Dans l'alchimie du «Nusseschnaps» la noix reste intacte: mais l'alcool, lui, est changé [...] Pourquoi n'ai-je pas tenté de m'exprimer en anglais, au cours de tant d'années vécues aux Etats-Unis ? C'est maintenant que je peux répondre à cette question : la macération de la noix alsacienne dans le whisky américain n'eût guère réussi. ${ }^{48}$

En d'autres termes : sur le tronc de l'anglais, la greffe du dialecte imprégné de la présence charnelle des choses et de leur saveur ne prend pas.

Quant à la seconde source retrouvée en lui-même qui lui permettra de donner vie à la langue, elle réside dans le mystère d'Israël. Au-delà ou en-deçà des images bibliques contribuant à détourner la langue et à l'extraire de son vêtement trop rigide, taillé dans le tissu de la rationalité, à l'aérer en quelque sorte, « tout se passe comme si le poète avait capté un mystère et, l'ayant enfoui au cœur de son inspiration, en suggérait la présence dans chaque vocable, dans chaque cadence, dans chaque idée ${ }^{49}$. Ce mystère, poursuit André Néher dans son évocation et son commentaire de La Corne du Grand Pardon, « c'est celui d'Israël [...], son mystère cosmique, celui-là même que la mystique juive, par un coup de sonde aux vibrations infinies, a décelé au centre du monde $»^{50}$. Le

\footnotetext{
${ }^{46}$ Ibid., p. 538.

${ }^{47}$ C. Vigée, S. Parizet, Les portes éclairées de la nuit, op. cit., p. 144.

${ }^{48}$ C. Vigée, La lune d'hiver, op. cit., p. 252-253.

49 André Néher, "À propos de La Corne du Grand Pardon » (texte paru pour la première fois dans la revue Évidences en novembre 1955), Peut-être n 4, 2013, p. 58. ${ }^{50}$ Ibid.
} 
poète ne s'inscrit-il pas dans la lignée d'Isaac Louria et des grands maîtres de Safed rassemblant les étincelles dispersées et emprisonnées dans les écorces du monde au lendemain de la catastrophe (la chevirah ou brisure des vases contenant la lumière divine, entraînant la dispersion et l'exil de Dieu) :

Exil de la parole, exil de la présence ;

Quand le printemps est absent le monde est en exil,

Chaque homme est en exil dans le désert du temps.

$[\ldots]$

Saurons-nous délivrer de l'enfer des gésines

La lumière égarée hors des vaisseaux rompus ? ${ }^{51}$

Quel autre témoignage de l'absence et de l'exil de Dieu que la terrible expérience de la Shoah? Pour le survivant, qui dédie La lune d'hiver à la mémoire de ses proches assassinés, aux «quarante-trois hommes, femmes et enfants, livrés aux nazis et brûlés dans les fours crématoires parce qu'ils étaient nés juifs en Alsace française ", la Corne $d u$ Grand Pardon sonnera également le rassemblement des enfants d'Israël dont les corps partirent en fumée :

Par les tessons de mots, qui luisent à travers

Les sombres alluvions du royaume des cendres,

L'énorme unité de la nuit est entamée. ${ }^{52}$

Lorsque paraît L'été indien, écrit dans lequel s'élabore la structure littéraire propre à l'œuvre du poète, le judan calqué sur le modèle biblique, mêlant l'horizontalité de la prose et la verticalité du poème, «contre-chant du roman classique trop concerté, et de la poésie désincarnée $\gg{ }^{53}$ caractéristiques de la tradition littéraire occidentale, Arnold Mandel, faisant écho à André Neher, note dans sa recension parue en mai 1955 dans le Bulletin des Communautés de Strasbourg: Vigée « parle 'juif' " 54 .

C'est donc avant même le " grand retour » à la terre originaire des ancêtres et la fécondation du français par l'hébreu, tant il est vrai qu'il se

\footnotetext{
51 «L'éclair de la présence », dans La Corne du Grand Pardon, MHT, op. cit., p. 208.

${ }^{52}$ Ibid., p. 164, « Échec à la nuit ».

${ }^{53}$ C. Vigée, S. Parizet, Les portes éclairées de la nuit, op. cit., p. 127.

54 « Arnold Mandel à propos de 1'Été indien », Peut-être n 4, 2013, op. cit., p. 61.
} 
renouvelle et s'alimente « au jeu des langues» qui l'encadrent ${ }^{55}$, que Claude Vigée relève le défi de «produire une littérature authentiquement juive » dans une langue doublement exilique, car langue du poème au sein de l'exil américain, et issue d'autre part d'une tradition ignorante de l'héritage judaïque et se refusant à la connaître ${ }^{56}$. Cette « réintégration du moi dans ses propres racines ignées ${ }^{57}$ au fil des années passées sous la lune d'hiver n'a toutefois pas valeur existentielle uniquement pour le poète, ainsi que l'a bien pressenti René Girard :

Les poèmes simples, puisant à la source, [...] ramènent le lecteur à « ses origines profondes », où qu'elles puissent se trouver [...] Le sentier qui mène au-dedans et celui qui débouche sur l'extérieur font une seule et même voie. ${ }^{58}$

\footnotetext{
${ }^{55}$ Voir à ce propos la contribution de Thierry Alcoloumbre, « 'Terre promise / Langue conquise'. Le problème de la langue chez Claude Vigée», dans L'œil témoin de la parole, op. cit., p. 131.

${ }^{56}$ Voir l'entretien avec Francine Kaufmann, dans La faille du regard, op. cit., p. 226 sqq.

${ }^{57}$ Claude Vigée, Le parfum et la cendre, Paris, Grasset, 1984, p. 119.

${ }^{58}$ René Girard, «Dans les blés étrangers 》 (texte paru en anglais dans Renascence, en été 1959), traduit par Anne Mounic (les termes en italiques figurent en français dans le texte original), Peut-être nº4, 2013, op. cit., p. 64-65.
} 